\title{
Green finance: Pandemic and climate change
}

\author{
Igor Klioutchnikov, Oleg Kliuchnikov* \\ ANO HE «IBI», Department of banking business and innovative financial technologies, 191011, Nevsky Ave. 60, S. Petersburg, Russia
}

\begin{abstract}
In the last decade, green finance has become an important area of tackling the environmental threats associated with climate change and a prerequisite for sustainable development. The Covid-19 outbreak has drawn additional attention to green finance as an economic mechanism for creating healthy living environments. The article examines the impact of COVID-19 on the financial industry, the participation of green finance in the economic recovery after the pandemic in the direction of considering the Paris Agreement on reducing greenhouse gas emissions. The authors put forward the provision on the existence of causal relationships between the "green" financial and "green" economy: "green" finance (reason) is a mechanism for the formation of a "green" economy (consequence). The impact of green finance on society can be greatly enhanced through changes in lifestyles, the behaviour of people and companies, legislative initiatives and government decisions aimed at protecting the health and the environment; climate change and the pandemic have increased the overall fragility of development and created additional risks that are factored into green finance. The article substantiates the position that the global pandemic will have a long-term impact on people's attitudes towards the environment and on the financing of this area. As uncertainty grows about protecting people from disease and mitigating climate change, green finance may become the mainstream of finance.
\end{abstract}

\section{Introduction}

Epidemics exploit the weaknesses of the societies in which they break out. The COVID-19 outbreak is no exception. It showed that the world is not ready to respond quickly to global threats related to climate change and pandemic, as well as to economic crises: most governments are slow to respond to changes, and their measures are poorly coordinated and not always effective. Lack of preparedness refers not only to dealing with pandemics, but also to prevent the irreversible effects of climate change and natural disasters [1]. The World Health Organisation (WHO) considers climate change to be the biggest global health threat in the 21 st century [2]. Air pollutants increase the risk of heart disease, chronic respiratory disease, stroke, and other diseases. It also increases the risk of dying from COVID-19. Overall, air pollution from fossil fuels is responsible for only 3.6 million premature deaths per year [3].

The global pandemic will have a long-term effect on human attitudes towards the environment and on the financing of this area. As uncertainties grow about protecting populations from disease and preventing climate change, it is important to build resilience to both COVID-19 and long-term environmental challenges. This can be done by focusing on green finance - increasing its efficiency and strengthening its position in post-pandemic economic recovery. This approach will yield significant short-term and long-term benefits and will make society more resilient to shocks over time.

In the last decade, green finance has become not only an important area of countering environmental threats and climate change but also a condition for sustainable development. They represent an attractive foundation for creating resource-efficient, less carbon, less harmful to the environment, more socially oriented, and inclusive communities. In modern conditions, environmental safety is becoming a decisive factor in sustainable development.

The authors put forward the assertion that there are causal relationships between the "green" financial and "green" economies: "green" finance (the cause) is the mechanism for the formation of the "green" economy (the consequence). In this paper, the authors make the following assumptions: (I) a narrow focus on postpandemic economic recovery may have adverse impacts on the environment and human health, as long-term impacts may not always be factored into short-term outcomes; (II) the impact of green finance on society can be significantly enhanced through changes in lifestyle, the behaviour of people and companies, legislative initiatives and government decisions aimed at respecting health and the environment; (III) climate change is linked both to the current pandemic (climate change has paved the way for the spread of some infectious diseases and weakened immunity) and to the priorities and behaviour of investors (in particular, it has accelerated the development of green finance); And (IV) climate change and the pandemic have increased overall development fragility and created

\footnotetext{
*orresponding author: okey003@mail.ru
} 
additional risks, which are reflected in green finance that is more resilient than traditional approaches.

\subsection{Background and related works}

Much of the economic literature on the optimal financial response to climate change has focused on the trade-off between direct costs and the potentially uncertain longterm benefits of investments to reduce carbon emissions [4]. At the same time, the discount rate and its parameters play a central role in this discussion, since even small changes in discount rates can dramatically change the current value of investments in long horizons. Thus, in the long term, make investments in reducing carbon emissions attractive or not attractive. In this regard, finance was considered from the standpoint of solving two problems: (I) climate change in the right direction, (II) adaptation of the production and household system to climate change. The impact of financial innovation on climate change is being actively studied/Policymakers and academics are just beginning to examine the scope and impact of COVID-19's impact on the financial industry and its involvement in post-pandemic economic recovery. However, the link between climate change and the current pandemic in light of the development of green finance is a new topic.

\section{Linking Climate Change to Pandemic and Green Finance}

The coronavirus pandemic could lead to a deeper understanding of the connections that bind us globally. Well-equipped health systems are essential to protect us from threats to health security, including climate change. Climate change has already made conditions more conducive to the spread of some infectious diseases, including Lyme disease, waterborne diseases, and mosquito-borne diseases, such as malaria and fever. Predicting future risks is not easy, but climate change is strongly affecting several areas that play a role in when and where pathogens appear, including temperature and rainfall. To limit the risk of infectious diseases, it is necessary to significantly reduce greenhouse gas emissions and limit global warming to 1.5 degrees. That is, to implement the Paris Agreement and step up green financing for economic development.

In the baseline scenario of post-pandemic development, considered by the experts of the World Economic Forum, the key condition for normalisation of activity includes investments in long-term human health, the environment, and a green economy [5]. Green finance plays a leading role in creating measures to preserve the environment and the sustainability of the economy, and in the new conditions in providing epidemiological measures to protect humans, which is extremely important for the effective reproduction of human capital. The green focus of post-pandemic economic recovery will increase the resilience of society to pandemics and other emergencies, including climate change [6]. In April 2020, at the initiative of the OECD General Secretariat, a paper was prepared to discuss post-pandemic economic recovery based on investments in environmental improvement.

Even small changes in the behaviour of people, companies, governments, and investors can have a significant impact on the state of green finance. As the events of 2019 and the first half of 2020 showed, prices for green bonds can fall without warning, or they can recover as quickly as they fell. While the short-term outlook for green bond investors is always uncertain, the medium to long-term outlook is encouraging. During the pandemic, in four months (mid-March - late July 2020), the stock market rose by almost $50 \%$ in terms of the S\&P 500. The growth was artificially induced. The current uncertainty is pushing investors to invest in technology companies, as well as in projects related to the environment and healthy lifestyles [7]

The cornerstone of green finance is leverage and investment to achieve projected hydrocarbon emissions. This approach refers to understanding them in a narrow sense. For example, a proposal to halve $\mathrm{CO} 2$ emissions by 2035 or a proposal by 3,500 leading economists in the world, including 27 Nobel Prize winners in economics and a coalition of 19 Fortune 100 companies, the world's 100 largest companies to introduce "carbon dividends"; the 1978 Kyoto Protocol and the 1987 Montreal Protocol for the Protection of the Stratospheric Ozone Layer as a continuation and development of the 1985 Vienna Convention for the Protection of the Ozone Layer. The unity of action in these processes was undermined by the United States, which in 2017 withdrew from the agreement and in 2019 made very controversial and controversial proposals.

Under the 1992 United Nations Framework Convention on Climate Change, countries have established international market mechanisms that provide an economic incentive to reduce emissions in countries without a climate target. The next generation of global carbon markets is based on Article 6 of the Paris Agreement. The world's largest carbon market is the United Nations Clean Development Mechanism under the Kyoto Protocol. However, the market did not lead to emission reductions, and projects financed under it were more often associated with violations of human rights and with issues of environmental destruction. In 2020, measures to block trade contacts led to a sharp drop in the carbon price under the Emissions Trading System (as of March 25, the price fell by almost $40 \%$ to a two-year low of just above 15 euros per tonne of $\mathrm{CO} 2$ [8]; it increased insignificantly). As a result, there has been an increased need to rethink the market mechanism for emissions regulation. The business suspension caused by the pandemic demonstrated both the possibility of reducing emissions and solving a number of other environmental problems associated with emissions into the atmosphere and the world's oceans.

In general, a market mechanism for regulating restrictions has been created. Emissions were regulated by means of a set of financial instruments. The carbon market has essentially become a financial market with its various instruments.

The dominant schools viewed the environment (especially the climate) and finance as independent 
systems. As soon as the modern pandemic began to unfold, the shortcomings of this approach were immediately revealed. It turned out that the environment is directly related to finance. It's not just a shift towards social distancing, which has affected the interaction of financial institutions with customers and contributed to the rapid development of digital services. Important changes in financial liquidity were outlined and financial assistance programs were deployed, and the financial system began to prepare for post-pandemic economic recovery.

Financial markets have usually been presented as an efficient system for allocating resources. It can be modelled and understood with general equilibrium models and representative agents behaving rationally to maximise profit. In this case, the prices are determined using, for example, the Black-Scholes-Merton formula. In essence, it all boiled down to a linear approach. At the same time, the optimisation of the predictability of results was carried out under the strict control of operators in the form of the state and the market. However, at first, during the 2008 crisis, the financial system showed its vulnerability. Internal misadventures turned out to be much more powerful in influencing the depth of shocks than externally. Over the past twelve years, trends have emerged that may indicate new threats to the system and they are not only a monstrous increase in debt and asymmetries but above all a gap in the balance between finance and the real sector. At the same time, the contradiction and the gap in the balance between financial and natural capital are highlighted. Climate change and environmental change have focused on leading actors who have the potential to impact the future of society. Among them, green finance occupies a special position. They can offer resources and a mechanism to ensure future sustainable development.

Using the Minsky Financial Instability Hypothesis (FIH) and Credit Cycle to account for cycle changes due to climate and pandemic threats sheds light on the irrationality of many traditional development finance methods. First of all, this approach allows us to distinguish between stable (hedging) and unstable (speculative) green finance for economic recovery, sustainable (EGS) and unsustainable development, reliable (ensuring healthy lifestyles and limiting environmental disasters), and unreliable (climatic threats; financial pyramids) growth. Applying Minsky's theory to green finance and its impact on sustainable and green development provides important insights for practitioners and policymakers, expanding the analysis of studies on stability and volatility, as well as uncertainty in the context of financial markets in the environmental direction.

A reorientation of the focus on investments in technology related to environmental protection rather than maximising growth and reducing costs once again indicates the direction of post-pandemic development. But it is about finding a balance in solutions to restore growth, sustainability, and green development. Investing in green technology may still be years of travel, but there must be a shift in thinking towards more flexible sprints a series of shorter, discrete efforts that demonstrate value along the way. All this is taking place against the background of a change in the business model in the real sector of production. Green finance is participating in its transformation into a green direction.

The pandemic primarily affected banks. The sudden decline in interest rates and customer behaviour as a result of market volatility required rapid analysis to support decisions such as revising lending strategies and deposit pricing. The effectiveness of these strategies directly affects the bank's ability to mitigate margin pressure in near-zero conditions. Measurements and instruments in many banks are not dynamic enough to meet adequate information needs. As a result, all the necessary data can be obtained. Buffer levels are eliminated, but not necessarily consistent. It appears that local governments, for example, will issue municipal bonds to finance green solutions at the local level.

The following main directions of the impact of climate risks on the financial business can be distinguished: (1) since the risk increases and its probabilistic assessments change, changes are expected in insurance, banking and stocks; (2) linear impacts are replaced by non-linear ones - socio-economic consequences propagate in a predominantly non-linear manner; (3) more and more hazards reach threshold values, beyond which physiological, anthropogenic or ecological systems are affected, then there is a danger for the operation of the entire financial system - the prospect of its failure; (4) temporary impacts, for example, associated with the postponement of the payback periods of investment projects and the increase in temporary asymmetries between expectations and actual restructuring; (5) geographical - climatic impacts can be both local and global in nature on the economy and require appropriate financial decisions; (6) systemic impacts - the direct impact of climate change on agriculture is local in nature, it can have indirect consequences for regions and sectors through interconnected socio-economic and financial systems; (7) the poorest communities and population groups are generally the most financially vulnerable to climate impacts.

\section{Green finance as a tool for the transition to a green economy}

The transition to a green economy is necessary. First of all, for the world to avoid catastrophic climate change. The International Energy Agency (IEA) estimates that additional investment in renewable energy and energy efficiency alone between 2015 and 2040 was the US \$ 26 trillion. Overall, reaching the global targets in 2030 may require mobilising green finance of $\$ 90$ trillion [9]. Under the influence of the pandemic, many problems in the economy and finance have worsened significantly. Some of these problems are temporary and will resolve quickly after the end of the pandemic, while others are long-term, but new ones have also emerged. If the change in the price of oil is a temporary problem, since there are no fundamental reasons for its low values, at least for the current decade, then investment in human capital becomes a decisive and new problem. It is broadly linked to 
countering negative climate change. It is widely believed that it is precisely the green focus of financial investment after the pandemic that will open up new prospects for sustainable growth, reduce the threat to society from climate change and create millions of additional jobs in the coming decades. [10] Big data finance analysts have observed that epidemiological methods of studying disease incidence and prevalence are well-suited for assessing financial risk and for building models that can analyse green inclusions in finance and economics. It turned out that, despite the differences in the subject area, there are general approaches for the analysis and predictive modelling of green finance and mass diseases and anti-epidemiological measures, as well as the risks associated with them. In addition, a significant part of the data used for analysis is common for these purposes. Modern epidemiological models of the spread of infection, as well as the stages of a pandemic and its exit from it, can affect the development of business and the market (its structure and even scale) and, thereby, direct investments in the appropriate direction [11]. Another use of data for green investment decisions will be information on industry rebounds and recovery rates, as the impact of COVID-19 on different industries varies significantly. The inclusion of current events that indicate changes, expand the possibilities for the preparation and operational revision of investment processes [12]. It can also enhance the impact of predictive models on decisionmakers, and with broad information about the results of forecasts and on-demand. Thus, the green focus of finance in the post-pandemic business cycle will to a certain extent become a predictive function.

Potential new pandemics, climate change, and their impacts around the world will threaten businesses in a variety of ways. For example, the frequency and intensity of extreme weather conditions can wreak havoc on factories, supply chains, and other infrastructure, and disrupt transportation. Drought will increase the cost of water, which is likely to affect the cost of raw materials and products. Climate variability can force companies to face uncertainty in resource prices for energy production, transportation, and insurance. And some products may become obsolete or lose their markets, such as equipment related to coal mining or skiing and recreation in areas where the snow will disappear. Covid-19 has suspended normal living standards. It changed lifestyles and had a profound impact on the environment. In an environment where all attention is focused on fighting viruses, climate problems are formally relegated to the background. Thus, during the pandemic, global $\mathrm{CO} 2$ emissions decreased and living conditions in large cities and industrial centres improved. This is an improvement in air quality due to a reduction in traffic. Satellite images show dramatic declines in air pollution in many countries, linked to the economic slowdown caused by the virus outbreak.

\section{Problems of monitoring green investment projects on the Internet}

In green finance, new values appear all the time, so it's crucial to handle them in a good way. Using the deep learning toolkit, it is possible to detect green embeddings into the flowing of investments. But how one can train this embedding for these new green values? This problem can be solved using machine learning, by including categorical features that represent objects of the real world, such as words, objects and categories. In this way, during inference, the browser can receive new object values that were not previously viewed. For this, it is necessary to carry out certain preparatory work, through which you can get a certain meaning from the input data.

It is also possible to highlight invisible values (for example, OOV (Out of Vocabulary) values. The challenge is to handle them correctly. Different algorithms have different methods for working with OOV values. Different assumptions about categorical features must also be considered differently. With deep learning applied to dynamic financial data as well as green case data, new values are constantly emerging. For the analysis, you can use the Tabula recommender system as an example. Some of the input that the model receives during inference contains invisible values, which is common in recommender systems. When working with models, it is important to set the item ID correctly. Item ID: each green indicator element (when investing or viewing orders) receives a unique identifier. This approach allows, in a dynamic environment, in which new proposals, applications, and investments are constantly received, to track according to certain criteria those who meet green financing. To handle the OOV, you must define its value. The problem with OOV values arises during the claim definition. Usually, the OOV value is associated with values not visible to the model during training. Therefore, to get the OOV value during inference, the model "does not know" what to do with it. A simple solution would be to replace all rare values with a special OOV token before starting the training. The point is that from the point of view of the model, all OOV values entering it are the same. Therefore, it is advisable to replace them with the OOV token during withdrawal. This approach achieves two positive results:

- The token is picked up during training. This allows you to select categorical traits and embed them in a token, which after training will be learned and stored for all OOV values.

- There will be a significant reduction in the risk that occurs when the token is adjusted to rare values. By examining the attachments of these values, you can teach the model how to use them, since they can be used to identify and explain features or random noise. This approach increases the level of confidence in the green identification of investment and incoming applications. Working with green OOV values is difficult. The proposed model uses the item identifier function to store different information for each item. This is similar to the pure co-filtration approach.

\section{Conclusion}

The world financial centres were the most affected by the pandemic, which affects the revaluation of green finance. Thus, green finance in academia and green finance in 
financial markets are very different concepts. In the financial industry, data deployment and collection is becoming key, and the only thing that matters are whether the financial product that real customers want can be delivered in sufficient green packaging and adequate liquidity.

The COVID-19 pandemic has become the biggest problem for financial institutions in nearly a century. As the economic impact spreads, financial institutions face some big priorities that require concrete steps to turn things around now as well as calibrate for the future. They are working to keep sales channels open, even though social distancing advice and oversight and compliance functions were never designed to work remotely. They try to manage revenues and customer expectations despite near-zero interest rates and growing pressure on consumers. And they need to keep an eye on brand strategy and issues that will shape their future, as market forces and consumer behaviour could change as a result of this crisis. There is a fairly wide range of assessments of the financial mechanisms for overcoming the crisis caused by the pandemic and the directions of financing the economic recovery [13]. However, the prevailing opinion is that recovery from the economic crisis caused by the pandemic will be based on sustainable development, there will be a transition to a new norm that considers the real threat to humanity, in particular from natural emergencies, primarily related to climate change [14]. Finance is designed to tackle the challenges of economic recovery in ways that help not only reduce risks and vulnerabilities to the economy but also reduce the emissions that cause climate change and increase development uncertainty.

\section{References}

1. C. Guerriero, A. Haines, M Pagano. Health and sustainability in post-pandemic economic policies. Nature Sustainability. 08 June. (2020)

2. Climate Change and Human Health, WHO, (2019).

3. J. Lelieveld. et al. Proc. Natl Acad. Sci. USA 116, 7192-7197. (2019)

4. S. Giglio, M. Maggiori, J. Stroebel, A. Weber. Climate Change and Long-Run Discount Rates: Evidence from Real Estate. Discussion paper series No. 10958, Sun, 29 november, (2015)

5. A Wyns. How our responses to climate change and the coronavirus are linked. World Economic Forum. 02 Apr. (2020)

6. A. Cox, and A. Piccolo, Environmental Health and strengthening resilience to pandemics, OECD. 21 April.p. 14. (2020)

7. S. Saurel. It Is Time to Open Your Eyes, the Stock Market Is in the Middle of a Tech Bubble. Medium, Jul 23. (2020)

8. J. C. Figueira, Green recovery could create over a million new jobs in the UK by 2050. Climate Action, 12 June. (2020)
9. X. Wang. How Data Scientists Can Train and Updates Models to Prepare for COVID-19 Recovery, KDnuggets, 29 April. (2020)

10. R Cho, How Climate Change Impacts the Economy. State of the Planet, June 20. (2019)

11. C. Watkiss. Coronavirus: The Immediate effect on climate change. Climate Action, 30 March. (2020)

12. E. Dans, We Have to Implement A Green Economic Recovery. Forbes, Jun 9. (2020)

13. Harvey, F. World health leaders urge green recovery from coronavirus crisis. The Guardian, 26 May. (2020)

14. E. Krukowska. European CEOs, Minsters Start Campaign for Green Recovery. Bloomberg Green, 14 April (2020) 